\title{
Emergence of methicillin-resistant Staphylococcus aureus (MRSA) ST8 in raw milk and traditional dairy products in the Tizi Ouzou area of Algeria
}

\author{
Y. Titouche,${ }^{1,2 *}$ A. Hakem, ${ }^{2}$ K. Houali, ${ }^{1}$ T. Meheut, ${ }^{3}$ N. Vingadassalon, ${ }^{3}$ L. Ruiz-Ripa, ${ }^{4}$ D. Salmi, ${ }^{1}$ A. Chergui, ${ }^{1}$ \\ N. Chenouf, ${ }^{2}$ J. A. Hennekinne, ${ }^{3}$ C. Torres, ${ }^{4}$ and F. Auvray ${ }^{3}$ \\ ${ }^{1}$ Laboratory of Analytical Biochemistry and Biotechnology, University of Mouloud Mammeri, BP17 RP15000, Tizi Ouzou, Algeria \\ ${ }^{2}$ Laboratory of Exploration and Valorization of Steppe Ecosystems, University of Ziane Achour, BP3117, Djelfa, Algeria \\ ${ }^{3}$ Université Paris Est, Anses, Laboratory for Food Safety, F-94700, Maisons-Alfort, France \\ ${ }^{4}$ Area Bioquímica y Biología Molecular, Universidad de La Rioja, Madre de Dios 51, 26006 Logrono, Spain
}

\section{ABSTRACT}

Staphylococcus aureus is one of the leading causes of food-borne illness worldwide. Raw milk and dairy products are often contaminated with enterotoxigenic strains of this bacterium. Some of these strains carry antimicrobial resistance, leading to a potential risk for consumers. The aim of this study was to characterize $S$. aureus strains circulating in raw milk and traditional dairy products for carriage of staphylococcal enterotoxin (se) genes and antimicrobial resistance. Overall, 62 out of 270 samples (23\%) were contaminated with S. aureus, and 69 S. aureus strains were identified. We studied the enterotoxin genes using 2 multiplex PCR targeting 11 se genes. Seventeen $(24.6 \%)$ isolates carried one or more genes encoding for staphylococcal enterotoxins. The most commonly detected se genes were seb and sep, followed by seh, sea, and see. Using the disk diffusion method, we found that resistance to penicillin $\mathrm{G}$ and tetracycline was the most common. Eleven isolates of methicillin-resistant $S$. aureus (MRSA) carried the mecA gene. All MRSA isolates belonged to the same spa type (t024) and sequence type (ST8), and carried the seb and sep enterotoxin genes. However, none of them carried the Panton Valentine leukocidin gene (lukF/S-PV). The presence of enterotoxigenic $S$. aureus strains, including MRSA, in raw milk and dairy products, raises a serious public health concern, because these strains may cause food poisoning outbreaks, be disseminated to the population, or both.

Key words: raw milk, dairy product, enterotoxin gene, Staphylococcus aureus, methicillin-resistant Staphylococcus aureus (MRSA)

Received December 25, 2018.

Accepted March 23, 2019.

*Corresponding author: yacinetitouche@yahoo.fr

\section{INTRODUCTION}

Staphylococcus aureus is a human and animal bacterial pathogen involved in multiple disease processes. It is the cause of a variety of diseases ranging in severity from slight skin infections to more severe diseases, such as pneumonia, endocarditis, osteomyelitis, septicemia, or toxic shock syndrome, among others (Lowy, 1998). This broad range of clinical syndromes results from a variety of extracellular components, including surface proteins, capsule, enzymes, and toxins (Lowy, 1998). S. aureus produces a wide array of toxins. Staphylococcal enterotoxins are a family of at least 23 serological types of heat-stable enterotoxins that belong to the larger family of pyrogenic toxin superantigens (Argudin et al., 2010; Kadariya et al., 2014; Zhang et al., 2018). Pyrogenic toxins cause superantigenic activity such as immunosuppression and nonspecific T-cell proliferation. A few hours after ingestion of food contaminated with staphylococcal enterotoxins, people experience intense diarrhea, vomiting, and abdominal pain. The emetic response in particular is characteristic of staphylococcal food poisoning (Le Loir et al., 2003; Hennekinne et al., 2012). Five staphylococcal enterotoxin types (A, B, C, D, and E) responsible for the symptoms of staphylococcal food poisoning were first described in the 1960s (Argudin et al., 2010). Since then, several other variants of staphylococcal enterotoxins or Staphylococcus-like toxins have been described (Argudin et al., 2010). Foods that have been frequently implicated in staphylococcal food poisoning are milk and cream, cream-filled pastries, butter, ham, cheeses, sausages, canned meat, salads, cooked meals, and sandwich fillings (Le Loir et al., 2003).

Antimicrobial agents are widely used in human and veterinary medicine, and also in animal husbandry and other agricultural activities (Kluytmans, 2010). They are administrated to animals to promote growth (in some countries), and to treat bacterial infections. However, excessive application of these agents has led to the 
emergence of resistant bacterial strains as a growing problem in developed countries (Jamali et al., 2015). In recent decades, the increasing prevalence of antimicrobial-resistant $S$. aureus is receiving widespread attention. Strains of methicillin-resistant S. aureus (MRSA) are of concern, given that they represent a significant cause of morbidity and mortality (Castro et al., 2016). Methicillin resistance is conferred by the presence of the mecA gene (or its homologue mecC) encoding for the production of an altered penicillin binding protein (PBP2a), with a low affinity for all $\beta$-lactam antimicrobials (Visciano et al., 2014).

In cattle, MRSA is an important cause of mastitis (Vanderhaeghen et al., 2010). Numerous reports have described the prevalence of MRSA in bovine milk and the transmission of MRSA between people and dairy cattle (Lee, 2003; Haran et al., 2012; Antoci et al., 2013; Caruso et al., 2016). The emerging problem of MRSA colonization and the links with human infection have an effect on both food production and on the health of people who work with animals, with possible risks of disease for the general population (Visciano et al., 2014). Recent studies have highlighted the potential role of food in the spread of MRSA lineages in humans (Oniciuc et al., 2017). Bulk tank milk has been identified as a source of MRSA, demonstrating the potential food safety risk for contaminated milk and dairy products entering the human food chain (Kreausukon et al., 2012). However, data on the prevalence of MRSA in food remain scarce. Surveillance and monitoring of MRSA in humans, food-producing animals, and food is therefore recommended.

Few data are available on the prevalence and antimicrobial susceptibility of $S$. aureus in food in Algeria. Further detailed investigations might provide useful information for the more efficient treatment of infections and a decrease in the use of antimicrobial agents. The objectives of this study were (1) to evaluate the prevalence of $S$. aureus in raw milk and traditional dairy products; (2) to determine the occurrence of enterotoxin genes; and (3) to create a snapshot of the antimicrobial resistance of $S$. aureus isolates in a designated area of Algeria.

\section{MATERIALS AND METHODS}

\section{Sample Collection}

Samples were collected during the dry periods (April to September) in 2 years (2014 and 2015). These samples included raw cow milk and traditional dairy products made from raw milk, collected at 4 levels (farms, collectors, dairy units, and market points) where milk was handled in the Tizi Ouzou area of Algeria. We collected samples from 25 dairy farms, 25 milk tanks, 5 dairy units, and 4 local markets. The farms were selected based on their membership in 5 different dairies (i.e., 5 farms for each dairy unit). The farms were located within a radius of $25 \mathrm{~km}$, in the regions of Freha and Azazga. The milk tanks were used to collect raw milk produced on the farms ( 5 milk tanks for each dairy unit). The market points were located in the city of Tizi Ouzou. Samples included raw milk (190) and traditional dairy products, including butter (24), cheese (3), and 2 acidified milks, rayeb (24) and l'ben (29). The small number of cheese samples was due to their availability during the sampling period. The traditional dairy products were produced with raw milk in factories located mostly in urban areas, and then distributed to market points. All samples were collected aseptically in sterile boxes, transferred immediately to the laboratory with ice packs, and analyzed within 1 to $2 \mathrm{~h}$ after sampling.

\section{S. aureus Isolation and Biochemical Identification}

To isolate $S$. aureus, $10 \mathrm{~mL}$ (raw milk) or $10 \mathrm{~g}$ (butter, cheese, or acidified milk) of each sample was added to $90 \mathrm{~mL}$ of buffered peptone water (Conda Pronadisa, Madrid, Spain) and homogenized. The resulting suspensions were diluted 1:10 in Giolitti Cantoni broth (Conda Pronadisa) supplemented with potassium tellurite (Pasteur Institute, Alger, Algeria) and incubated for enrichment at $37^{\circ} \mathrm{C}$ for $24 \mathrm{~h}$. A loopful of each enriched broth was then streaked onto mannitol salt agar (Conda Pronadisa). The plates were incubated for 24 to $48 \mathrm{~h}$ at $37^{\circ} \mathrm{C}$ and observed for bacterial growth. Then, 1 or 2 suspected colonies (depending on their macroscopic structure and their charge in agar plates) were subcultured onto brain heart infusion agar (Biokar, Beauvais, France) to obtain pure cultures. These were submitted to Gram stain to confirm coccus morphology. The identification of isolates was completed using the following biochemical tests: fermentation of mannitol, catalase test, coagulase test, DNase (thermonuclease) test, and Voges-Proskauer test for acetoin production. The 2 reference strains used for microbiological analysis were $S$. aureus ATCC 25923 and $S$. aureus ATCC 43300. After identification, all isolates were stored in brain heart infusion broth (Biokar) with glycerol (30\% $\mathrm{vol} / \mathrm{vol}$ ) at $-20^{\circ} \mathrm{C}$ for further analysis.

\section{Molecular Characterization of S. aureus Isolates}

DNA Extraction. Strains were grown overnight in brain heart infusion broth at $37^{\circ} \mathrm{C}$ and streaked onto a milk plate count agar (Bio-Rad, Marnes la Coquette, France) incubated at $37^{\circ} \mathrm{C}$ for $24 \mathrm{~h}$, before DNA extraction. DNA was extracted using the InstaGene Kit 
(Bio-Rad) according to the manufacturer's recommendations (http://www.bio-rad.com/webroot/web/pdf/ lsr/literature/LIT544.pdf). We adjusted DNA concentrations $100 \mathrm{ng} / \mu \mathrm{L}$ using a Nanodrop 1000 spectrophotometer (Thermo Scientific, Wilmington, DE).

Identification of $S$. aureus Isolates by $P C R$ Amplification of the 23S rRNA Gene. We performed PCR simplex tests to detect the presence of a $23 \mathrm{~S}$ ribosomal RNA gene region specific for $S$. aureus according to Straub et al. (1999). The 1250-bp-long PCR products were electrophoresed in a $2 \%$ agarose gel and visualized by ethidium bromide staining $(1 \mu \mathrm{g} /$ $\mathrm{mL}$ ) using the Gel Doc EQ apparatus (Bio-Rad). A 1-kb DNA ladder (Promega, Lyon, France) was used as a molecular weight standard. The reference strain FRI361 was used as a positive control.

Detection of Enterotoxin Genes by Multiplex $P C R$. Two multiplex PCR assays were used to detect se genes. The first reaction was performed with 6 primer pairs and allowed for the detection of sea, seb, sec, sed, see, and ser genes. The second reaction was performed with 5 primer pairs and allowed for the detection of seg, seh, sei, sej, and sep genes. We performed PCR amplification and electrophoresis of PCR products as described previously (Roussel et al., 2015) according to the method developed and validated by the European Union Reference Laboratory for Coagulase Positive Staphylococci (EURL CPS). We used 5 reference S. aureus strains (FRIS6, 374F, FRI137, FRI326, and FRI361) as positive controls.

Detection of the mecA, mecC, spa, and lukS/ F-PV Genes. A multiplex PCR was performed as described by Stegger et al. (2012), with modifications, to detect mecA (162 pb), mecC (138 pb), Panton Valentine toxin (lukF/S-PV; $85 \mathrm{pb})$, and spa (200-600 pb). A 1-kb DNA ladder (Promega) was used as a molecular weight standard. Three references strains were used as positive controls: $S$. aureus LGA251 (carrying the mecC gene), S. aureus ATCC 25923 (carrying the pvl gene), and $S$. aureus MU50 (carrying the mecA gene).

Characterization of MRSA Strains by spa Typing, Multilocus Sequence Typing, and PulsedField Gel Electrophoresis. The polymorphic X-region of the protein A gene (spa) was amplified by PCR using primers spa-1113f (5'-TAAAGACGATCCTTCGGTGAGC-3') and spa-1514r (5'-CAGCAGTAGTGCCGTTTGCTT-3') (Aires de Sousa et al., 2006). The PCR products were electrophoresed in a $2 \%$ agarose gel and visualized using the Gel Doc EQ apparatus (Bio-Rad). They were further sequenced by Eurofins (Esberg, Germany), on both DNA strands. We determined spa types from the resulting DNA sequences using BioNumerics version 7.1 (Applied Maths, SintMartens-Latem, Belgium).
We performed multilocus sequence typing in MRSA strains as previously described (Lozano et al., 2012): we obtained the allelic profile of each isolate by sequencing internal fragments of 7 housekeeping genes ( $\operatorname{arc} C$, aroE, $g l p F, g m K, p t a, t p i$, and yqiL), allowing us to determine the sequence type, using the multilocus sequence typing database (http://saureus.mlst.net/).

Pulsed-field gel electrophoresis was performed using SmaI as the restriction enzyme, according to the EURL CPS method (Roussel et al., 2015).

\section{Antimicrobial Susceptibility of S. aureus Isolates}

The disk diffusion method was used to examine the antimicrobial resistance of $S$. aureus isolates on Mueller-Hinton agar (Biokar) according to the guidelines of the Clinical and Laboratory Standards Institute (CLSI, 2018). The antibiotic disks from Liofilchem (Roeseto, Italy) are listed as follows (antibiotic concentration in $\mu \mathrm{g}$, unless otherwise specified): penicillin G (10 IU), cefoxitin (30), gentamicin (10), amikacin (30), kanamycin (30), tobramycin (10), neomycin (30), tetracycline (30), erythromycin (15), spiramycin (100), lincomycin (15), ofloxacin (15), norfloxacin (15), clindamycin (2), chloramphenicol (30), trimethoprim/sulfamethoxazole (1.25/23.75), fosfomycin (50), fusidic acid (10), novobiocin (30), and bacitracin (10 IU). We used $S$. aureus ATCC 25923 in susceptibility testing as a control strain.

The minimal inhibitory concentration (MIC) of oxacillin was determined by using the agar dilution method (CLSI, 2018) for S. aureus isolates that showed cefoxitin resistance by disk diffusion. We determined MIC in Mueller-Hinton agar (Biokar) plus $2 \% \mathrm{NaCl}$ (wt/vol; Scharlau, Barcelona, Spain). We used S. aureus ATCC 29213 as a control. Phenotypic detection of MRSA was performed by cefoxitin disk diffusion test or by oxacillin MIC determination.

\section{Detection of Antimicrobial Resistance Genes and the scn Gene of the Immune-Evasion Cluster in MRSA Strains}

We used PCR to determine the presence of the tet $(\mathrm{K})$, tet $(\mathrm{M})$ and $\operatorname{tet}(\mathrm{L})$ genes, which confer resistance to tetracycline, and the bla $Z$ gene, which confers resistance to penicillin (Lozano et al., 2012). All MRSA strains were tested by PCR for the scn gene, marker of the immune evasion cluster system (Lozano et al., 2012).

\section{RESULTS}

\section{Prevalence of S. aureus}

Of 270 samples of raw milk and traditional dairy products made from raw milk, 62 were contaminated 
Table 1. Occurrence of Staphylococcus aureus in milk and traditional dairy products

\begin{tabular}{llcc}
\hline Product & $\begin{array}{l}\text { Sampling } \\
\text { point }\end{array}$ & $\begin{array}{c}\text { No. of } \\
\text { samples }\end{array}$ & $\begin{array}{c}\text { No. of positive } \\
\text { samples (\%) }\end{array}$ \\
\hline Raw milk & Farms & 75 & $13(17.3)$ \\
& Milk tanks & 75 & $18(24)$ \\
& Dairy units & 5 & $2(40)$ \\
Butter & Market points & 35 & $18(51.4)$ \\
Cheese & Market points & 24 & $0(0)$ \\
Rayeb & Market points & 3 & $0(0)$ \\
L'ben & Market points & 24 & $4(16.7)$ \\
Total & Market points & 29 & $7(24.1)$ \\
\hline
\end{tabular}

with S. aureus (23\%; Table 1). The frequency of $S$. aureus contamination varied by the type of product and also by the sampling point: it was higher in raw milk $(19.9 \%)$ than in milk products $(13.8 \%)$, and higher in milk tanks (24\%) and market points $(25.2 \%)$ than in farms $(17.3 \%)$. A total of $51(18.9 \%)$ raw milk samples and $11(4.1 \%)$ acidified raw milk samples were contaminated; we observed no contamination with $S$. aureus for butter and cheese samples made from raw milk.

We obtained a total of $69 \mathrm{~S}$. aureus isolates from the 62 positive samples [61 isolates from raw milk and 8 from acidified milk (l'ben or rayeb)]. One S. aureus isolate was retrieved from 55 samples, and 2 isolates from the remaining 7 samples.

\section{Occurrence of Enterotoxin Genes}

Of the 69 recovered isolates, $17(24.6 \%)$ carried at least 1 gene encoding for staphylococcal enterotoxin [15 isolates from raw milk and the remaining 2 from acidified milk (l'ben)]. Overall, we detected 5 se genes (i.e., sea, seb, see, seh, and sep). Four isolates carried only 1 gene (sea, see, seh, or sep), and 13 carried 2 genes (seb and sep). None of the isolates contained the sec or sed genes.

\section{Antimicrobial Resistance of S. aureus Isolates}

The highest resistance rates were to penicillin G $(91.3 \%)$ and tetracycline (47.8\%; Table 2). All isolates were susceptible to gentamicin, amikacin, chloramphenicol, sulfamethoxazole/trimethoprim, and novobiocin. However, we observed low resistance rates to cefoxitin/oxacillin (15.9\%), ofloxacin/norfloxacin (15.9\%), erythromycin (2.9\%), spiramycin (1.4\%), lincomycin/ clindamycin $(1.4 \%)$, tobramycin $(2.9 \%)$, and kanamycin (1.4\%). Sixteen S. aureus isolates $(23.18 \%)$ were resistant to at least 3 different classes of antimicrobial agents, and we observed 6 phenotypes of multidrug resistance (Table 3).
Table 2. Antimicrobial resistance of Staphylococcus aureus strains isolated from milk and dairy products $(\mathrm{n}=69)$

\begin{tabular}{lc}
\hline Antimicrobial agent & $\begin{array}{c}\text { No. }(\%) \text { of } \\
\text { resistant strains }\end{array}$ \\
\hline Penicillin G & $63(91.3)$ \\
Cefoxitin & $11(15.9)$ \\
Oxacillin & $11(15.9)$ \\
Gentamicin & $0(0)$ \\
Amikacin & $0(0)$ \\
Kanamycin & $1(1.4)$ \\
Neomycin & $1(1.4)$ \\
Tobramycin & $2(2.9)$ \\
Erythromycin & $2(2.9)$ \\
Spiramycin & $1(1.4)$ \\
Lincomycin & $1(1.4)$ \\
Tetracycline & $33(47.8)$ \\
Clindamycin & $1(1.4)$ \\
Chloramphenicol & $0(0)$ \\
Ofloxacin & $11(15.9)$ \\
Norfloxacin & $11(15.9)$ \\
Sulfamethoxazole/trimethoprim & $0(0)$ \\
Fosfomycin & $1(1.4)$ \\
Novobiocin & $0(0)$ \\
Bacitracin & $3(4.3)$ \\
Fusidic acid & $1(1.4)$ \\
\hline
\end{tabular}

\section{Detection and Characterization of MRSA}

We identified 11 MRSA strains (cefoxitin/oxacillinresistant), and they harbored the mecA gene, representing a low prevalence among collected samples (4.1\%). Nine strains were isolated from raw milk and 2 from acidified milk (l'ben), obtained from 2 collectors, 1 farm and 3 local markets (Figure 1). None of the MRSA strains carried the Panton Valentine toxin (lukF/S-PV) gene. Typing of the MRSA strains showed that they all belonged to the same spa type (t024), sequence type (ST8), and pulsed-field gel electrophoresis pulsotype; they harbored the blaZ and tetK genes (encoding for penicillin and tetracycline resistance, respectively) and enterotoxin genes, as well as the scn gene (immune eva-

Table 3. Phenotypic resistance patterns among multidrug-resistant Staphylococcus aureus strains tested $(\mathrm{n}=69)$

\begin{tabular}{lcc}
\hline $\begin{array}{l}\text { Antimicrobial resistance } \\
\text { phenotype }^{1}\end{array}$ & $\begin{array}{c}\text { No. of isolates with } \\
\text { phenotype (\% with } \\
\text { respect to S. aureus) }\end{array}$ & $\begin{array}{c}\text { mecA } \\
\text { gene }\end{array}$ \\
\hline P-FOX-OX-TE-OFX-NOR & $11(15.9)$ & + \\
P-TE-K-N-FC & $1(1.4)$ & - \\
P-E-CD-MY-SP & $1(1.4)$ & - \\
P-TE-TOB-BA & $1(1.4)$ & - \\
P-FOS-BA & $1(1.4)$ & - \\
P-TE-TOB & $1(1.4)$ & - \\
Total & $16(23.2)$ & - \\
\hline
\end{tabular}

${ }^{1} \mathrm{BA}=$ bacitracin $\mathrm{CD}=$ clindamycin $; \mathrm{E}=$ erythromycin $; \mathrm{FC}=$ fusidic acid; $\mathrm{FOS}=$ fosfomycin; $\mathrm{FOX}=$ cefoxitin; $\mathrm{K}=$ kanamycin; $\mathrm{MY}=$ lincomycin; $\mathrm{N}=$ neomycin; $\mathrm{NOR}=$ norfloxacin; $\mathrm{OFX}=$ ofloxacin; $\mathrm{OX}=$ oxacillin $; \mathrm{P}=$ penicillin $; \mathrm{SP}=$ spiramycin $; \mathrm{TE}=$ tetracycline; $\mathrm{TOB}=$ tobramycin 
sion cluster system), indicating their clonal relationship and epidemiological link.

\section{DISCUSSION}

In our study, the $S$. aureus contamination rate of raw milk was lower than previously observed in Algeria by Adjalne-Kaouche et al. (2014); these authors reported a prevalence of 33.3 and $56.7 \%$ in raw milk samples collected at farms and dairy units, respectively. Moreover, Chaalal et al. (2018) described a $S$. aureus prevalence of 32.6 and $9.1 \%$ in raw milk and pasteurized milk samples, respectively. A high prevalence of $S$. aureus in raw milk and dairy products has also been reported in other countries (Kamal et al., 2013; Jamali et al., 2015; Rola et al., 2015; Giaciniti et al., 2017; Papadopoulos et al., 2018). The contamination of dairy products by $S$. aureus might have been caused by the contamination of raw milk used for production, but also by crosscontamination during the manufacturing process. Cows with mastitis represented the major source of $S$. aureus contamination of milk and dairy products, as demonstrated by others (Kümmel et al., 2016).

Our results differ from those reported elsewhere for raw milk and dairy products, in which a higher prevalence of enterotoxigenic S. aureus was observed (Mo-

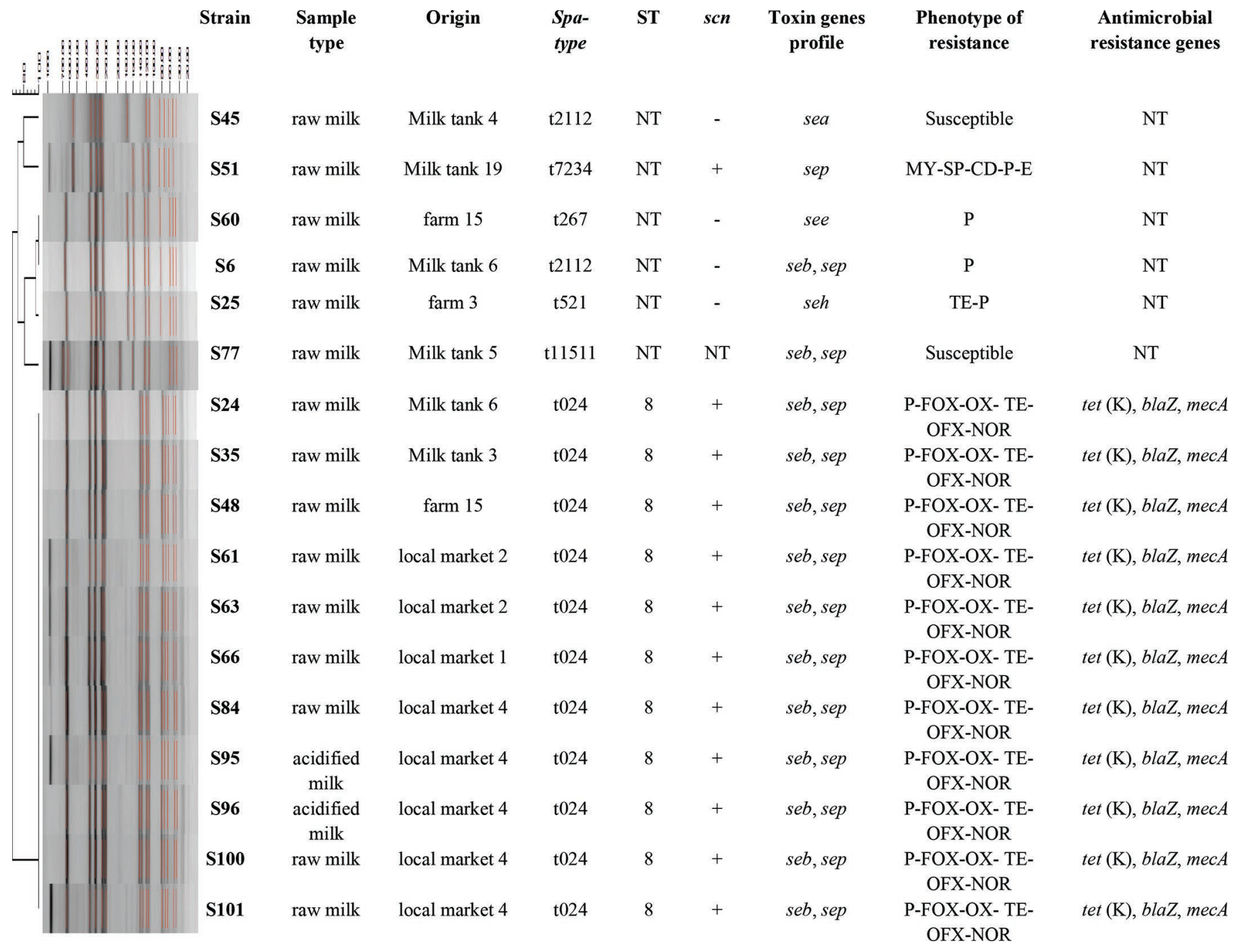

Figure 1. Pulsed-field gel electrophoresis analysis of the 17 enterotoxin gene-positive Staphylococcus aureus strains recovered from raw milk and acidified milk, using the SmaI restriction enzyme. Only bands with a high-intensity peak were assigned, marked with red lines, and considered for comparison of patterns. High-molecular-weight bands outside the range of the reference band sizes were not considered. We obtained a dendrogram of similarity using the Dice coefficient and the unweighted-pair group method, using arithmetic averages with $1 \%$ optimization and $1 \%$ tolerance. $\mathrm{NT}=$ not tested; $\mathrm{ST}=$ sequence type; $\mathrm{CD}=$ clindamycin; $\mathrm{E}=$ erythromycin; $\mathrm{FOX}=$ cefoxitin; $\mathrm{MY}=$ lincomycin; $\mathrm{NOR}=$ norfloxacin; $\mathrm{OFX}=$ ofloxacin; $\mathrm{OX}=$ oxacillin; $\mathrm{P}=$ penicillin; $\mathrm{SP}=$ spiramycin; $\mathrm{TE}=$ tetracycline 
randi et al., 2007; Rall et al., 2008; Mehli et al., 2017). Although sec and sed are the most reported enterotoxin genes in food, we did not observe them in our study. In contrast, seb and sep were the most prevalent enterotoxin genes in our study, detected in almost all isolates. The staphylococcal enterotoxins most commonly involved in cases of staphylococcal food poisoning are A and D, followed by B. Staphylococcal enterotoxin C has been recognized as an important cause of staphylococcal food poisoning associated with the consumption of dairy products (Normanno et al., 2007).

We identified a high resistance rate to penicillin and tetracycline. Similar results have been observed for strains isolated from milk, dairy products, and dairy workers (Tan et al., 2014; Visciano et al., 2014; Jamali et al., 2015; Castro et al., 2016; Chaalal et al., 2018; Papadopoulos et al., 2018). Increasing resistance levels are known to derive from the use of antibiotics in various sectors, such as human communities, hospitals, farms, and companion animals (Davies and Davies, 2010; Gonzalez-Candelas et al., 2017). All isolates were susceptible to gentamicin, amikacin, chloramphenicol, sulfamethoxazole/trimethoprim, and novobiocin. The lack of resistance to chloramphenicol, an antibiotic prohibited in the European Union for animals intended for human consumption, contrasts with reports from many countries (Pereira et al., 2009; Tan et al., 2014; Castro et al., 2016). However, residues of chloramphenicol detected in animal tissues in Poland may suggest its use in veterinary practice (EFSA, 2014). The absence of or slight resistance to aminoglycosides (i.e., gentamicin, kanamycin, and tobramycin) that we observed agrees with previous studies (Yesim-Can and Haluk-Celik, 2012; Jamali et al., 2015; Rola et al., 2015). Jamali et al. (2015) reported a low resistance to erythromycin and lincomycin for strains isolated from bovine milk, sheep, and traditional cheeses. A low resistance to fluoroquinolone (ciprofloxacin) was observed by Shahraz et al. (2012) for strains isolated from hamburger. Results of our cefoxitin disk diffusion test were in concordance with the PCR-based detection of the mecA gene, so this method was very suitable for the detection of MRSA. Several studies have found cefoxitin to be superior to oxacillin in detecting mecA-mediated resistance, especially for $S$. aureus strains with low-level resistance. (Felten et al., 2002; Hung et al., 2011).

In our survey, the prevalence of MRSA was low. Only a few studies have been conducted in Algeria to assess the prevalence of MRSA in food, providing little information on the genetic types of the strains isolated. Chaalal et al. (2014) reported a prevalence of $3.5 \%$ in raw milk and meat, and Akkou et al. (2015) isolated 4 MRSA strains from workers in contact with animals. A study conducted by Chaalal et al. (2018) revealed an MRSA prevalence of $21.5 \%$ in various food products, including raw milk and meat. Studies from Malaysia, Egypt, and Turkey have reported prevalence of 2, 5.3, and $4.7 \%$, respectively (Aras et al., 2012; Kamal et al., 2013; Jamali et al., 2015). A study in Minnesota dairy farms found 2 MRSA-positive samples out of 150 pooled bulk tank milk samples (Haran et al., 2012). In Italy, the frequency of MRSA isolation from milk and dairy products differed between studies, which might reflect the heterogeneity of the methods used and factors such as the type of tested samples (geographical origin, manufacturing technology, use of pasteurized vs. raw milk, sample storage and handling). Six MRSA strains $(0.4 \%)$ were isolated from 1634 milk and cheese samples (Normanno et al., 2007), and prevalences ranging from 0.7 to 1.2 and $8.3 \%$ were reported in other studies (Basanisi et al., 2015; Carfora et al., 2015; Cortimiglia et al., 2015; Caruso et al., 2016; Basanisi et al., 2017; Giaciniti et al., 2017). In Greece, 11 samples (3\%) of milk and dairy products were contaminated by MRSA carrying the mecA gene (Papadopoulos et al., 2018).

All 11 MRSA isolates in our study were resistant to antimicrobial agents other than $\beta$-lactams, such as fluoroquinolones and tetracycline, indicating a multidrug-resistant phenotype, as in other studies (Haran et al., 2012; Kreausukon et al., 2012; Parisi et al., 2016; Basanisi et al., 2017; Papadopoulos et al., 2018); nevertheless, none of the MRSA strains carried the Panton Valentine toxin (lukF/S-PV) gene, an important virulent factor associated with pathogenicity. This contrasted with other studies, where pvl-positive MRSA was obtained from bovine milk and raw milk in Algeria (Chaalal et al., 2018), Korea (Kwon et al., 2005), and China (Wang et al., 2014); from bulk tank milk in the United States (Haran et al., 2012) and Italy (Mancini et al., 2015; Basanisi et al., 2017); and from raw milk fresh cheese in Colombia (Herrera et al., 2016).

The presence of se genes in MRSA isolates contaminating raw milk has been previously reported (Normanno et al., 2007; Haran et al., 2012; Parisi et al., 2016). However, few data are available on the occurrence of MRSA in staphylococcal food poisoning. Jones et al. (2002) described the first report of an outbreak of gastrointestinal illness caused by community-acquired MRSA, and Kérouanton et al. (2007) identified 2 MRSA out of 33 S. aureus strains recovered from staphylococcal food poisoning. Transmission of MRSA to humans through contact with farm animals has been well documented. The first zoonotic transmission episodes from pig to human were reported in the last decade in the Netherlands (Voss et al., 2005). Since then, the prevalence of CC398 MRSA in food-producing animals has increased, 
and people in direct contact with live animals are at higher risk of colonization or infection than the general population (EFSA, 2009). In contrast, information about the potential transmission of MRSA to humans through the food chain remains limited (Petinaki and Spiliopoulou, 2012), although several studies indicate that food handling and consumption may play a role in MRSA spread and transmission to humans (Kluytmans et al., 1995; Jones et al., 2002; Oniciuc et al., 2017). In some cases of food transmission, the MRSA isolates were identified as hospital-associated MRSA or community-associated MRSA, indicating that food handlers were likely to be the source of food contamination. In other surveys, livestock-associated MRSA strains were the primary isolates, indicating an animal source of food contamination (Doulgeraki et al., 2017).

In our study, genetic typing revealed that strains isolated from the different sampling points (farms, milk tanks, and market points) belonged to the same sequence type (ST8), spa type (t024), and pulsed-field gel electrophoresis pulsotype, suggesting that strains circulating at farms may be found at the end of the dairy chain, but it is difficult to confirm the origin of these strains because the nasal carriage of animals and farmers, which constitute the main source of $S$. aureus at the farm level, were not checked. Genetic similarity among the $S$. aureus isolates collected in a larger geographical region has also been observed in studies performed on bulk milk from cows, sheep, and goats (Vautor et al., 2003, 2009; Mørk et al., 2005; Haran et al., 2012; Antonios et al., 2015; McMillan et al., 2016; Mehli et al., 2017). Staphylococcus aureus is known to cause contagious mastitis (Kümmel et al., 2016), and sharing of strains within and between herds has been observed for dairy cows and sheep, even over long distances, suggesting the existence of special properties in the dominant strains, including special virulenceassociated genes (Vautor et al., 2003). The ST8 lineage has been detected in MRSA isolates of raw milk fresh cheese in Colombia; this sequence type, related to the USA 300 type, is the predominant cause of communityassociated MRSA infection in the United States, which has been disseminated in South America, particularly in Colombia (Herrera et al., 2016). Other spa types have been identified for MRSA isolated from milk and foodstuffs throughout the world, such as clonal complex CC398-associated t011 and t034 spa types for bovine milk MRSA in Germany (Kreausukon et al., 2012). In Italy, a high genetic diversity has been observed in MRSA strains isolated from milk and dairy products, with most spa types identified belonging to clonal complex CC398 (Carfora et al., 2015; Cortimiglia et al., 2015; Traversa et al., 2015; Caruso et al., 2016).

\section{CONCLUSIONS}

The raw milk and dairy products analyzed in this study had risk factors associated with their consumption because of the high presence of $S$. aureus, confirming them as vehicles for the transmission of potential pathogenic bacteria. The association of enterotoxigenic and multidrug-resistant properties in $S$. aureus isolates points out a serious public health risk, because enterotoxins might be present in food and MRSA could be disseminated to the population. Spread to other countries may also occur through global animal and food trading, or through international travel of healthy carriers. Continuous monitoring and further improvement of the hygienic quality of raw milk and dairy products is necessary. For this, the application of good manufacturing practices and hazard analysis and critical control point (HACCP) systems are crucial for ensuring the microbial safety and quality of food products.

\section{ACKNOWLEDGMENTS}

We thank S. Granier (Anses, Laboratory for Food Safety) for a helpful discussion on MRSA characterization, and for experimental procedures. This research did not receive any specific grants from funding agencies in the public, commercial, or not-for-profit sectors. The group of the University of La Rioja (Spain) was financed by project SAF2016-76571-R of the Agencia Estatal de Investigación (AEI) of Spain and FEDER.

\section{REFERENCES}

Adjalne-Kaouche, S., R. Benhacine, F. Ghozlane, and A. Mati. 2014. Nutritional and hygienic quality of raw milk in the mid-northern region of Algeria: Correlations and risk factors. Scientific World J. 2014:131593.

Aires-de-Sousa, M., K. Boye, H. DeLencastre, A. Deplano, M. C. Enright, J. Etienne, A. Friedrich, D. Harmsen, A. Holmes, X. W. Huijsdens, A. M. Kearns, A. Mellmann, H. Meugnier, J. K. Rasheed, E. Spalburg, B. Strommenger, M. J. Struelens, F. C. Tenover, J. Thomas, U. Vogel, H. Westh, J. Xu, and W. Witte. 2006. High interlaboratory reproducibility of DNA sequence-based typing of bacteria in a multicenter study. J. Clin. Microbiol. 44:619-621.

Akkou, M., K. Antri, M. A. Bachtarzi, M. Bes, A. Tristan, O. Dauwalder, R. Kaidi, H. Meugnier, M. Tazir, J. Etienne, F. Laurent, and N. Ramdani-Bouguessa. 2015. Phenotypic and genotypic characterization of Staphylococcus aureus strains associated with bovine mastitis and nasal carriage of workers in contact to animals in Algeria. Pak. Vet. J. 36:184-188.

Antoci, E., M. R. Pinzone, G. Nunnari, S. Stefani, and B. Cacopardo. 2013. Prevalence and molecular characteristics of methicillin-resistant Staphylococcus aureus (MRSA) among subjects working on bovine dairy farms. Infez. Med. 21:125-129.

Antonios, Z., P. Theofilos, M. Ioannis, S. Georgios, V. Georgios, B. Evridiki, E. Loukia, M. Kyriaki, A. Athanasios, and L. Vasiliki. 2015. Prevalence, genetic diversity, and antimicrobial susceptibility profiles of Staphylococcus aureus isolated from bulk tank milk from Greek traditional ovine farms. Small Rumin. Res. 125:120-126. 
Aras, Z., I. Aydin, and K. Kav. 2012. Isolation of methicillin-resistant Staphylococcus aureus from caprine mastitis cases. Small Rumin. Res. 102:68-73.

Argudin, M. Á., M. C. Mendoza, and M. R. Rodicio. 2010. Food poisoning and Staphylococcus aureus enterotoxins. Toxins (Basel) $2: 1751-1773$.

Basanisi, M. G., G. Nobili, G. La Bella, R. Russo, G. Spano, G. Normanno, and G. La Salandra. 2015. Molecular characterization of Staphylococcus aureus isolated from sheep and goat cheeses in Southern Italy. Small Rumin. Res. 135:17-19.

Basanisi, M. G., G. La Bella, G. Nobili, I. Franconieri, and G. La Salandra. 2017. Genotyping of methicillin-resistant Staphylococcus aureus (MRSA) isolated from milk and dairy products in South Italy. Food Microbiol. 62:141-146.

Carfora, V., A. Caprioli, N. Marri, D. Sagrafoli, C. Boselli, G. Giaciniti, G. Giangolini, L. Sorbara, S. Dottarelli, A. Battisti, and S. Amatise. 2015. Enterotoxin genes, enterotoxin production, and methicillin resistance in Staphylococcus aureus isolated from milk and dairy products in Central Italy. Int. Dairy J. 42:12-15.

Caruso, M., L. Latorre, G. Santagada, R. Fraccalvieri, A. Miccolupo, R. Sottili, L. Palazzo, and A. Parisi. 2016. Methicillin-resistant Staphylococcus aureus (MRSA) in sheep and goat bulk tank milk from Southern Italy. Small Rumin. Res. 135:26-31.

Castro, A., C. Santos, H. Meireles, J. Silva, and P. Teixeira. 2016. Food handlers as potential sources of dissemination of virulent strains of Staphylococcus aureus in the community. J. Infect. Public Health 9:153-160.

Chaalal, W., H. Aggad, N. Saidi, and M. Kihal. 2014. Prevalence and antimicrobial resistance of methicillin-resistant Staphylococcus aureus isolated from raw meat and milk in Algeria. Adv. Environ. Biol. 8:1-5.

Chaalal, W., N. Chaalal, N. Bourafa, M. Kihal, S. M. Diene, and J. M. Rolain. 2018. Characterization of Staphylococcus aureus isolated from food products in Western Algeria. Foodborne Pathog. Dis. 15:353-360.

CLSI (Clinical and Laboratory Standard Institute). 2018. Performance Standards for Antimicrobial Susceptibility Testing. 28th ed. Suppl. M100. CLSI, Wayne, PA.

Cortimiglia, C., V. Bianchini, A. Franco, A. Caprioli, A. Battisti, L. Colombo, K. Stradiotto, F. Vezzoli, and M. Luini. 2015. Prevalence of Staphylococcus aureus in bulk tank milk from dairy goat farms in Northern Italy. J. Dairy Sci. 98:2307-2311.

Davies, J., and D. Davies. 2010. Origins and evolution of antibiotic resistance. Microbiol. Mol. Biol. Rev. 74:417-433.

Doulgeraki, A. I., P. Di Ciccio, A. Ianieri, and G. E. Nychas. 2017. Methicillin-resistant food-related Staphylococcus aureus: A review of current knowledge and biofilm formation for future studies and applications. Res. Microbiol. 168:1-15.

EFSA (European Food Safety Authority). 2009. Assessment of the public health significance of methicillin resistant Staphylococcus aureus (MRSA) in animals and foods. EFSA J. 993:1-73.

EFSA (European Food Safety Authority). 2014. Report for 2012 on the Results From the Monitoring of Veterinary Medicinal Products Residues and Other Substances in Live and Animal Products. EFSA Supporting Publication 2014: EN-540. EFSA, Parma, Italy.

Felten, A., B. Grandry, P. H. Lagrange, and I. Casin. 2002. Evaluation of three techniques for detection of low-level methicillin-resistant Staphylococcus aureus (MRSA): a disk diffusion method with cefoxitin and moxalactam, the Vitek 2 system, and the MRSAscreen latex agglutination test. J. Clin. Microbiol. 40:2766-2771.

Giaciniti, G., V. Carfora, A. Capriol, D. Sagrafoli, N. Marri, G. Giangolini, R. Amoruso, M. Lurescia, F. Stravino, S. Dottarelli, F. Feltrin, A. Franco, S. Amatiste, and A. Battisti. 2017. Prevalence and characterization of methicillin-resistant Staphylococcus aureus carrying mecA or mecC and methicillin-susceptible Staphylococcus aureus in dairy sheep farms in central Italy. J. Dairy Sci. 100:7857-7863.

Gonzalez-Candelas, F., I. Comas, J. L. Martinez, J. C. Galan, and F. Baquero. 2017. The evolution of antibiotic resistance. Pages 257-284 in Genetics and Evolution of Infectious Diseases. 2nd ed. M. Tibayrenc, ed. Elsevier, Amsterdam, the Netherlands.
Haran, K. P., S. M. Godden, D. Boxrud, S. Jawahir, J. B. Bender, and S. Sreevatsan. 2012. Prevalence and characterization of Staphylococcus aureus, including methicillin-resistant Staphylococcus aureus, isolated from bulk tank milk from Minnesota dairy farms. J. Clin. Microbiol. 50:688-695.

Hennekinne, J. A., M. L. De Buyser, and S. Dragacci. 2012. Staphylococcus aureus and its food poisoning toxins: Characterization and outbreak investigation. FEMS Microbiol. Rev. 36:815-836.

Herrera, F. C., M. L. G. Lopez, and J. A. Santos. 2016. Short communication: Characterization of methicillin-resistant Staphylococcus aureus isolated from raw milk fresh cheese in Colombia. J. Dairy Sci. 99:7872-7876.

Hung, K. H., J. J. Yan, Y. C. Lu, H. M. Chen, and J. J. Wu. 2011. Evaluation of discrepancies between oxacillin and cefoxitin disk susceptibility for detecting methicillin-resistant Staphylococcus aureus. Eur. J. Clin. Microbiol. Infect. Dis. 30:1181-1184.

Jamali, H., M. Paydar, B. Radmenhr, S. Ismail, and A. Dadrasnia. 2015. Prevalence and antimicrobial resistance of Staphylococcus aureus isolated from raw milk and dairy products. Food Control 54:383-388.

Jones, T. F., M. E. Kellum, S. S. Porter, M. Bell, and W. Schaffner. 2002. An outbreak of community-acquired foodborne illness caused by methicillin-resistant Staphylococcus aureus. Emerg. Infect. Dis. 8:82-84.

Kadariya, J., T. C. Smith, and D. Thapaliya. 2014. Staphylococcus aureus and staphylococcal food-borne disease: An ongoing challenge in public health. BioMed Res. Int. 2014:827965.

Kamal, R. M., M. A. Bayoumi, and S. F. A. Abd El Aal. 2013. MRSA detection in raw milk; some dairy products and hands of dairy workers in Egypt, a mini-survey. Food Control 33:49-53.

Kérouanton, A., J. A. Hennekinne, C. Letertre, L. Petit, O. Chesneau, A. Brisabois, and M. L. De Buyser. 2007. Characterization of Staphylococcus aureus strains associated with food poisoning outbreaks in France. Int. J. Food Microbiol. 115:369-375.

Kluytmans, J., W. Van Leeuwen, W. Goessens, R. Hollis, S. Messer, L. Herwaldt, H. Bruining, M. Heck, J. Rost, and N. van Leeuwen. 1995. Food-initiated outbreak of methicillin-resistant Staphylococcus aureus analyzed by pheno- and genotyping. J. Clin. Microbiol. $33: 1121-1128$

Kluytmans, J. A. 2010. Methicillin-resistant Staphylococcus aureus in food products: Cause for concern or case for complacency? Clin. Microbiol. Infect. 16:11-15.

Kreausukon, K., A. Fetsch, B. Kraushaar, K. Alt, K. Muller, V. Kromker, Z. H. Zessin, A. Kasbohrer, and B. A. Tenhagen. 2012. Prevalence, antimicrobial resistance, and molecular characterization of methicillin-resistant Staphylococcus aureus from bulk tank milk of dairy herds. J. Dairy Sci. 95:4382-4388.

Kümmel, J., B. Stessl, M. Gonano, G. Walcher, O. Bereuter, M. Fricker, T. Grunert, M. Wagner, and M. Ehling-Schulz. 2016. Staphylococcus aureus entrance into the dairy chain: Tracking $S$. aureus from dairy cow to cheese. Front. Microbiol. 7:1603.

Kwon, N. H., K. T. Park, J. S. Moon, W. K. Jung, S. H. Kim, J. M. Kim, S. K. Hong, H. C. Koo, Y. S. Joo, and Y. H. Park. 2005. Staphylococcal cassette chromosome mec (SCCmec) characterization and molecular analysis for methicillin-resistant Staphylococcus aureus and novel SCCmec subtype Ivg isolated from bovine milk in Korea. J. Antimicrob. Chemother. 56:624-632.

Le Loir, Y., F. Baron, and M. Gautier. 2003. Staphylococcus aureus and food poisoning. Genet. Mol. Res. 2:63-76.

Lee, J. H. 2003. Methicillin (oxacillin)-resistant Staphylococcus aureus strains isolated from major food animals and their potential transmission to humans. Appl. Environ. Microbiol. 69:6489-6494.

Lowy, F. D. 1998. Staphylococcus aureus infections. N. Engl. J. Med. 339:520-532.

Lozano, C., A. Rezusta, P. Gómez, E. Gómez-Sanz, N. Báez, G. Martin-Saco, M. Zarazaga, and C. Torres. 2012. High prevalence of spa types associated with the clonal lineage CC398 among tetracycline-resistant methicillin-resistant Staphylococcus aureus strains in a Spanish hospital. J. Antimicrob. Chemother. 67:330-334. 
Mancini, F., M. G. Basanisi, and A. Pantosti. 2015. An unusual PVLpositive MRSA strain in milk and dairy products from region of South Italy. J. Glob. Antimicrob. Resist. 3:151-152.

McMillan, K., S. C. Moore, C. M. McAuley, N. Fegan, and E. M. Fox. 2016. Characterization of Staphylococcus aureus isolates from raw milk sources in Victoria, Australia. BMC Microbiol. 16:169.

Mehli, L., S. Hoel, B. G. M. Thomassen, A. N. Jakobsen, and H. Karlsen. 2017. The prevalence, genetic diversity and antibiotic resistance of Staphylococcus aureus in milk, whey, and cheese from artisan farm dairies. Int. Dairy J. 65:20-27.

Morandi, S., M. Brasca, R. Lodi, P. Cremonesi, and B. Castiglioni. 2007. Detection of classical enterotoxins and identification of enterotoxin genes in Staphylococcus aureus from milk and dairy products. Vet. Microbiol. 124:66-72.

Mørk, T., T. Tollersrud, B. Kvitle, H. J. Jorgensen, and S. Waage. 2005. Comparison of Staphylococcus aureus genotypes recovered from cases of bovine, ovine, and caprine mastitis. J. Clin. Microbiol. 43:3979-3984

Normanno, G., M. Corrente, G. La Salandra, A. Dambrosio, N. C. Quaglia, A. Parisi, G. Greco, A. L. Bellacicco, S. Virgilio, and G. V. Celano. 2007. Methicillin-resistant Staphylococcus aureus (MRSA) in foods of animal origin product in Italy. Int. J. Food Microbiol. 117:219-222.

Oniciuc, E. A., A. I. Nicolau, M. Hernandez, and D. Rodriguez-Lazaro. 2017. Presence of methicillin Staphylococcus aureus in the food chain. Trends. Food Sci. Technol. 61:49-59.

Papadopoulos, P., T. Papadopoulos, A. S. Angelidis, E. Boukouvala, A. Zdragas, A. Papa, C. Hadjichristodoulou, and D. Sergelidis. 2018. Prevalence of Staphylococcus aureus and methicillin-resistant S. aureus (MRSA) along the production chain of dairy products in north-western Greece. Food Microbiol. 69:43-50.

Parisi, A., M. Caruso, G. Normanno, L. Latorre, R. Sottili, A. Miccolupo, R. Fraccalvieri, and G. Santagada. 2016. Prevalence, antimicrobial susceptibility and molecular typing of methicillin-resistant Staphylococcus aureus (MRSA) in bulk tank milk from Southern Italy. Food Microbiol. 58:36-42.

Pereira, V., C. Lopes, A. Castro, J. Silva, P. Gibbs, and P. Teixeira. 2009. Characterization for enterotoxin production, virulence factors, and antibiotic susceptibility of Staphylococcus aureus isolates from various foods in Portugal. Food Microbiol. 26:278-282.

Petinaki, E., and I. Spiliopoulou. 2012. Methicillin-resistant Staphylococcus aureus among companion and food chain animals: impacts of human contacts. Clin. Microbiol. Infect. 18:626-634.

Rall, V. L., F. P. Vieira, R. Rall, R. L. Vieitis, A. Fernandes Jr, J. M. Candeias, K. F. Cardoso, and J. P. Araújo Jr.. 2008. PCR detection of staphylococcal enterotoxin genes in Staphylococcus aureus strains isolated from raw and pasteurized milk. Vet. Microbiol. 132:408-413.

Rola, J. G., A. Korpysa-Dzirba, A. Czubkowska, and J. Osek. 2015. Prevalence of enterotoxin genes and antimicrobial resistance of coagulase-positive staphylococci recovered from cow milk. J. Dairy Sci. 98:4273-4278.

Roussel, S., B. Felix, N. Vingadassalon, J. Grout, J. A. Hennekinne, L. Guillier, A. Brisabois, and F. Auvray. 2015. Staphylococcus aureus strains associated with food poisoning outbreaks in France: Comparison of different molecular typing methods, including MLVA. Front. Microbiol. 6:882.

Shahraz, F., H. Dadkhah, R. Khaksar, M. Mahmoudzadedh, H. Hosseini, M. Kamran, and P. Bourke. 2012. Analysis of antibiotic resistance patterns and detection of mecA gene in Staphylococcus aureus isolated from packaged hamburger. Meat Sci. 90:759-763.

Stegger, M., P. S. Andersen, A. Kearns, B. Pichon, M. A. Holmes, G Edwards, F. Laurent, C. Teale, R. Skov, and A. R. Larsen. 2012. Rapid detection, differentiation and typing of methicillin-resistant Staphylococcus aureus harbouring either mecA or the new mecA homologue mecA (LGA251). Clin. Microbiol. Infect. 18:395-400.

Straub, J. A., C. Hertel, and W. P. Hammes. 1999. A 23S rDNAtargeted polymerase chain reaction-based system for detection of Staphylococcus aureus in meat starter culture and dairy products. J. Food Prot. 62:1150-1156.

Tan, S. L., H. Y. Lee, and N. A. Mahyudin. 2014. Antimicrobial resistance of Escherichia coli and Staphylococcus aureus isolated from food handler's hands. Food Control 44:203-207.

Traversa, A., G. R. Gariano, S. Gallina, D. M. Bianchi, R. Orusa, L. Domenis, P. Cavallerio, L. Fossati, R. Serra, and L. Decastelli. 2015. Methicillin resistance in Staphylococcus aureus strains isolated from food and wild animal carcasses in Italy. Food Microbiol. $52: 154-158$

Vanderhaeghen, W., K. Hermans, F. Haesebrouck, and P. Butaye. 2010. Methicillin resistant Staphylococcus aureus (MRSA) in food production animals. Epidemiol. Infect. 138:606-625.

Vautor, E., G. Abadie, J. M. Guibert, C. Huard, and M. Pépin. 2003. Genotyping of Staphylococcus aureus isolated from various sites of farms with dairy sheep using pulsed-field gel electrophoresis. Vet. Microbiol. 96:69-79.

Vautor, E., V. Magnone, G. Rios, K. Le Brigand, D. Bergonier, G. Lina, H. Meugnier, P. Barbry, R. Thiéry, and M. Pépin. 2009. Genetic differences among Staphylococcus aureus isolates from dairy ruminant species: A single-dye DNA microarray approach. Vet. Microbiol. 133:105-114.

Visciano, P., F. Pomilio, R. Tofalo, L. Sacchini, M. A. Saletti, E. Tieri, M. Schirone, and G. Suzzi. 2014. Detection of methicillinresistant Staphylococcus aureus in dairy cow farms. Food Control $46: 532-538$.

Voss, A., F. Loeffen, J. Bakker, C. Klaassen, and M. Wulf. 2005. Methicillin-resistant Staphylococcus aureus in pig farming. Emerg. Infect. Dis. 11:1965-1966.

Wang, X., G. Li, X. Xia, B. Yang, M. Xi, and J. Meng. 2014. Antimicrobial susceptibility and molecular typing of methicillin-resistant Staphylococcus aureus in retails foods in Shaanxi, China. Foodborne Pathog. Dis. 11:281-286.

Yesim Can, H., and T. Haluk Celik. 2012. Detection of enterotoxigenic and antimicrobial resistant $S$. aureus in Turkish cheeses. Food Control 24:100-103.

Zhang, D. F., X. Y. Yang, J. Zhang, X. Qin, X. Huang, Y. Cui, M. Zhou, C. Shi, N. P. French, and X. Shi. 2018. Identification and characterization of two novel superantigens among Staphylococcus aureus complex. Int. J. Med. Microbiol. 308:436-446. 